\title{
The Effects of Corrosion, Fatigue, and Corrosion-fatigue of Multilayer Coated Polyesters for Flexible Electronics Applications
}

\author{
Dilveen Mohammed, ${ }^{\mathrm{a}, \mathrm{b}, \dagger}$ Rayan Ameen, ${ }^{\mathrm{a}, \mathrm{b}}$ Steven Street, ${ }^{\mathrm{b}}$ Kostas Sierros, ${ }^{\mathrm{c}}$ James Bowen, ${ }^{\mathrm{d}}$ \\ Stephen Kukureka ${ }^{b}$ \\ ${ }^{a}$ University of Duhok, Department of Physics, Zakho way, Duhok, Iraq \\ ${ }^{\mathrm{b}}$ University of Birmingham, School of Metallurgy and Materials, Edgbaston, Birmingham, B15 2TT, United Kingdom \\ c West Virginia University, Department of Mechanical \& Aerospace Engineering, Morgantown, West Virginia, 26506, USA \\ ${ }^{\mathrm{d}}$ Open University, Department of Engineering and Innovation, Walton Hall, Milton Keynes MK7 6AA, United Kingdom \\ †Corresponding author:Dilveen@uod.ac,Dilveenmohammed83@gmail.com
}

Received: 13 March, 2021; Accepted: 23 May, 2021; Published: 19 June, 2021

We investigated the electrical and optical properties of ITO/Ag-alloy/ITO films in a corrosive $\mathrm{NaCl}$ solution. The effects of bending fatigue combined with aggressive environments provided by the salt solution were also studied. It is shown that exposure to the salt solution can cause the ITO/Ag-alloy/ITO performance to degrade over time. In addition, greater changes in electrical resistance and lower values of optical transmittance were observed at higher concentrations of the salt solution. The combination of stress and corrosion by the aqueous $\mathrm{NaCl}$ solution was found to significantly reduce the conductivity of the ITO/Ag-alloy/ITO film. Furthermore, it is shown that the pres-

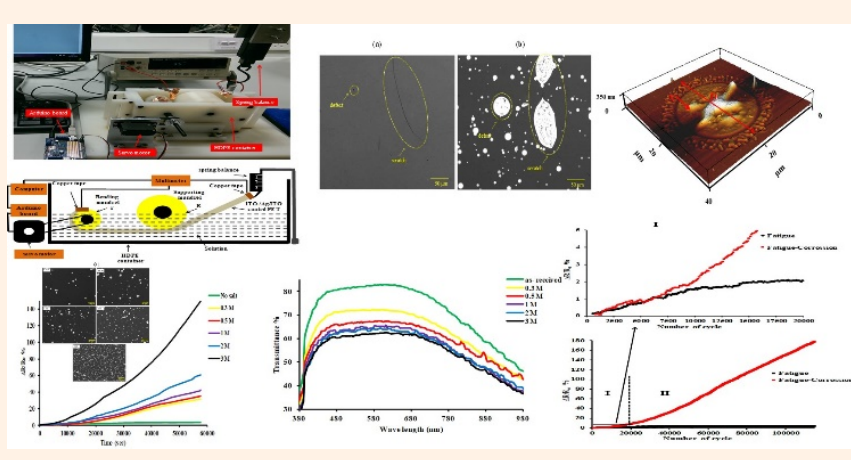
ence of structural defects in the deposited films can provide a driving force to form Ag agglomerations that, in turn, degrade the conductivity of the ITO/Ag-alloy/ITO film. This suggests that defects in the film should be minimized to avoid excessive corrosion.

Keywords ITO/Ag-alloy/ITO; Polymer substrates; PET; Agglomeration

\section{INTRODUCTION}

High optical transmittance $(>90 \%)$ and low electrical resistivity $\sim 7 \times 10^{-4}(\Omega \mathrm{cm})$ [1] make indium tin oxide (ITO) a good candidate anode material for optoelectronic devices such as solar cells, touch panels, liquid crystal displays, and organic light-emitting diodes [2, 3]. However, two drawbacks of ITO limit its application in flexible electronics: (1) the brittle nature of ITO make it susceptible to cracking and delamination when it is under mechanical deformation during manufacturing and in service conductions [4], and (2) scarceness of indium ore results in high prices for ITO and contributes to the reduction of the quantities employed for film fabrication. It is difficult to reduce the thickness to under $150 \mathrm{~nm}$ in the case of single ITO films because of the resulting, significant, decrease in electrical conductance [5]. However, a very thin metal film can be deposited between two ITO films to result in a higher conductivity than that of a single ITO layer of the same thickness [5] and to improve the mechanical properties due to the ductile metallic interlayer [6]. Such a multilayer system has been proved to be effective in achieving electrically conductive and optically transparent electrodes by optimizing the thickness of the ITO layer [7]. Ag is a commonly used interlayer since it has resistivity comparable to other metals such as $\mathrm{Cu}$ [8]. Ag has also been alloyed with a variety of elements including $\mathrm{Cu}$, $\mathrm{Au}$, and $\mathrm{Pd}$, which can improve the stability to heat and moisture of the multilayer film structure [9].

Because of the wide application of flexible electronic devices, it is expected that such devices may be required to 
effectively function upon significant exposure to a harsh outdoor environment. A prime example is solar cell panel installations in challenging climates. The long-term stability of solar energy is still limited by the instability of Ag/Ag-alloy-based transparent conducting oxide (TCO) electrodes which are very sensitive to the presence of reactants in the surrounding atmosphere such as chlorine, acids, and ozone [10] as well as to exposure to high temperatures and moisture environments [11]. Chlorine atoms which exist in the ambient atmosphere particularly in marine environments can cause corrosion and degradation of the multilayer films after exposure to the atmosphere for long periods of time. Also, an additional cause of corrosion can be photolithographic patterning processes for thin films that involve roll-to-roll etching in $\mathrm{HCl}$ solutions [12-14], leaving residual $\mathrm{Cl}$ ions in the multilayer.

Few studies have reported on the corrosion of Ag/Ag-alloy-based TCO deposited on a polymer substrate. Nadel et al. [14] found film cracking, peeling of the ZnO top layer, and agglomeration using scanning electron microscopy (SEM) after $24 \mathrm{~h}$ of exposure of $\mathrm{ZnO} / \mathrm{Ag} / \mathrm{ZnO} /$ glass to water containing sodium chloride. The agglomerated material was analyzed by energy dispersive X-ray spectroscopy (EDS) in SEM and was identified as being rich in Ag. They concluded that the high level of the chlorine concentration which may have been introduced from fingerprints or other direct contamination that can have caused corrosion through cracking and delamination of the top $\mathrm{ZnO}$ layer.

Koike et al. [15] investigated the durability of the multilayer sputter coating including silver layer as the intermediate layer. After immersion in the $0.1-\mathrm{M}$ sodium chloride solution for $17 \mathrm{~h}$, white spots were found on the surface of the multilayer sputter coating resulting from the migration of $\mathrm{Ag}$ atoms from the $\mathrm{Ag}$ layer. To improve the corrosion resistance of the multilayer coatings, they also sputtered $0.5-2 \mathrm{~nm}$ thick transition metals, such as Ti, V, Ta, and $\mathrm{Zr}$, on the top surface of the multilayer coatings, and then the appearance, electrical resistance, and optical transmittance of the multilayer coatings were evaluated. They observed that durability against salt-water exposure was dramatically improved by deposition of Ti and Ta to the top surface of the multilayer (ITO/Ag/ITO/Ag/ITO/Ag/ITO/PET substrate) without a significant decrease in transmittance.

Chen et al. [16] reported that many corrosion spots appear when an ITO/Ag/ITO/glass multilayer is exposed for $144 \mathrm{~h}$ to $323 \mathrm{~K}$ with a relative humidity of $90 \%$, and that these spots are related to the corrosion of $\mathrm{Ag}$. However, there are no spots observed on the surface of ITO/AgTi/ITO due to the improved corrosion resistance of the Ag-Ti alloy as compared to Ag. It is also observed that the conductivity and the optical transmittance of ITO/Ag/ITO at $550 \mathrm{~nm}$ wavelength were significantly decreased after the durability test but no change was found for ITO/AgTi/ITO. They concluded that ITO/AgTi/ITO was a promising candidate for transparent conducting electrodes.

In addition to degradation when subjected to a corrosive environment, transparent electrodes may fail when subjected to the combination of mechanical stress and corrosion that they may encounter during manufacture and/or use, ultimately leading to stress corrosion cracking.

A few other studies have paid attention to the effect of combination of stress and corrosion on transparent conducting electrodes. Sierros et al. [17] stated that, in such a combined mechano-chemical environment, the primary cause of premature failure of ITO is stress corrosion cracking. They wrapped ITO-coated polyethylene terephthalate (PET) specimen around mandrels with various diameters and then submerged them in a corrosive environment provided by acrylic acid. It was found that the combination of both applied stress and corrosion can cause cracking to occur at strains below a quarter of those needed for ITO electrical failure with no corrosion. It was also noted that the corrosion is observed to form in rough specimen areas thus suggesting that substrates with smooth surfaces are important for avoiding extreme corrosion.

In this work, we investigated the change in electrical resistance of ITO/Ag-alloy/ITO films as a function of time in the presence of a $\mathrm{NaCl}$ aqueous solution. In addition, the optical transmittance of the ITO/Ag-alloy/ITO film deposited on flexible PET after exposure to the aqueous salt for 16 $\mathrm{h}$ was measured. The latter is an important consideration for a number of applications such as displays and solar cells. Furthermore, the effects of bending fatigue combined with aggressive and corrosive environments provided by the salt were studied.

\section{EXPERIMENTAL DETAILS}

\section{A. Full immersion corrosion experiments}

ITO ( 95 nm)/Ag-alloy $(\sim 10 \mathrm{~nm}) /$ ITO $(\sim 95 \mathrm{~nm})$ films deposited on PET substrates $(125 \mu \mathrm{m}$ thick) by a commercial roll-to-roll magnetron sputtering machine (CSEM Brasil) were used in this study. The pristine samples exhibited a sheet resistance of $11 \Omega \mathrm{sq}^{-1}$. Five concentrations of $\mathrm{NaCl}$ solutions $(0.3,0.5,1,2$, and $3 \mathrm{M})$ were prepared from analytical grade sodium chloride (Sigma-Aldrich and Fisher Scientific) with deionized water. The resulting solutions were used as the corrosive medium during testing. Corrosion measurements were conducted with specimens of $\sim 1 \mathrm{~cm}$ in width and $\sim 22 \mathrm{~cm}$ in length. The specimens were cut from the ITO/Ag-alloy/ITO sputtered sheets. The samples were cleaned using distilled water and dried in air before performing the experiment. Then, the sample edges were clamped using crocodile clips inside an open plastic container. Afterwards, the container was filled with the $\mathrm{NaCl}$ solution and left for 16000 and $57600 \mathrm{~s}$ at room temperature and the change in electrical resistance was monitored in situ using a multimeter (Fluke 45). The concentration range was chosen because seawater has chloride concentration of about $0.6 \mathrm{M}$ [18]. Special care had to be paid to prevent stressing the sample when clamping it inside the container using a copper conductive tape for optimizing the contact between the sample surface and the clips. After immersion, the sam- 
ples were removed from the $\mathrm{NaCl}$ solution, quickly washed using distilled water, and subsequently dried in air.

\section{B. Corrosion-fatigue experiments}

Corrosion-fatigue experiments were performed using a custom-built cyclic uniaxial bending apparatus based on that used by Bejitual et al. [19]. A schematic diagram and the setup of this apparatus is shown in Figure 1. The sample was wrapped over a mandrel made from chlorinated polyvinyl chloride (c-PVC) on one end and it was fixed to a spring balance at the other end. The copper conductive tape was used at each end of the sample to allow optimized electrical contact for resistance measurements. A large c-PVC mandrel $(R \gg r)$ supported the sample in the horizontal position and prevented sagging of the sample. A servomotor was utilized to rotate the mandrel with the reciprocating rotary motion controlled by a board driven using the Arduino software. The tests were run at a fixed reciprocating frequency of $2 \mathrm{~Hz}$ Each test was carried out for 115200 cycles. The response of the film in terms of the electrical resistance was monitored in situ using the multimeter (Fluke 45).

A high-density polyethylene (HDPE) container was filled with $0.3 \mathrm{M}$ of the $\mathrm{NaCl}$ solution for the corrosion-fatigue test. Before and after testing, the samples were washed with distilled water and dried using compressed air. The data obtained through this test are the number of cycles to failure with the corresponding electrical resistance changes. Note that the ITO/Ag-alloy/ITO surface is placed under tension. According to the mandrel radius $r$ of $12.5 \mathrm{~mm}$, sample strips of approximately $202 \mathrm{~mm}$ in length and $10 \mathrm{~mm}$ wide were cut from the sputtered sheets. The bending tensile stain, $\varepsilon$, from this mandrel was calculated to be $0.5 \%$ using the equa-
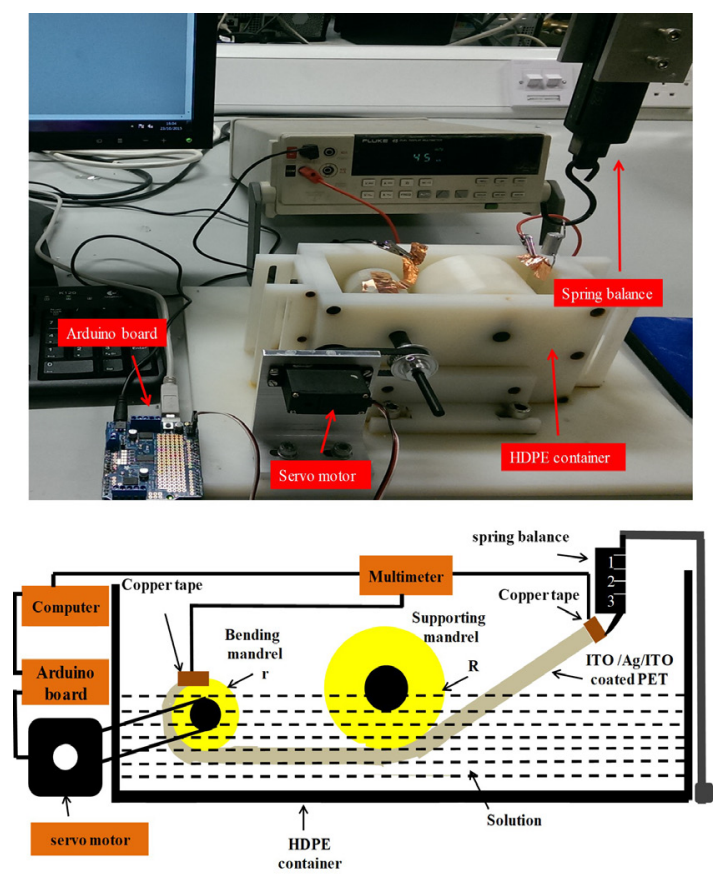

Figure 1: Photograph and schematic diagram of the custom-built bending fatigue apparatus. tion [20]:

$$
\varepsilon=\frac{t_{\mathrm{s}}+t_{\mathrm{f}}}{2 r}
$$

where $t_{\mathrm{s}}$ and $t_{\mathrm{f}}$ are substrate and film thicknesses, respectively.

\section{Surface analysis}

Sample surfaces were studied using confocal laser scanning microscopy (CLSM) and SEM. Prior to the SEM observation, the samples were sputtered with gold of approximately $5 \mathrm{~nm}$ thick to dissipate electric charging. EDS was also utilized to conduct film elemental analysis. Surface topography changes were examined using an atomic force microscope (AFM) operating in contact-mode. Change in the optical transmittance of the specimen was investigated by using a spectrophotometer (Jenway 6310) in the region of 300-1000 nm.

\section{RESULTS AND DISCUSSION}

\section{A. Corrosive degradation of ITO/Ag-alloy/ITO}

In this experiment, the impact of aggressive aqueous $\mathrm{NaCl}$ environments on the ITO/Ag/ITO film strips was investigated. The specimens were cleaned by distilled water and dried by compressed air and were put into a bath of 0.3 , $0.5,1,2$, and $3 \mathrm{M}$ of the $\mathrm{NaCl}$ solution for $57600 \mathrm{~s}(16 \mathrm{~h})$, and the electrical resistance was monitored in situ to assess the reliability of the conducting films.

Figure 2 shows the normalized change in electrical resistance over time for the ITO/Ag-alloy/ITO films along with the as-received films immersed in different concentrations of $\mathrm{NaCl}$. In general, the results obtained from this investigation indicate that, when the ITO/Ag-alloy/ITO film is exposed to $\mathrm{NaCl}$, a loss of the electrical function of the film over time will be caused by agglomeration of Ag. In particular, for the samples in the $0.3-$ and $0.5-\mathrm{M}$ solutions, the per cent increase in the electrical resistance is still close to zero

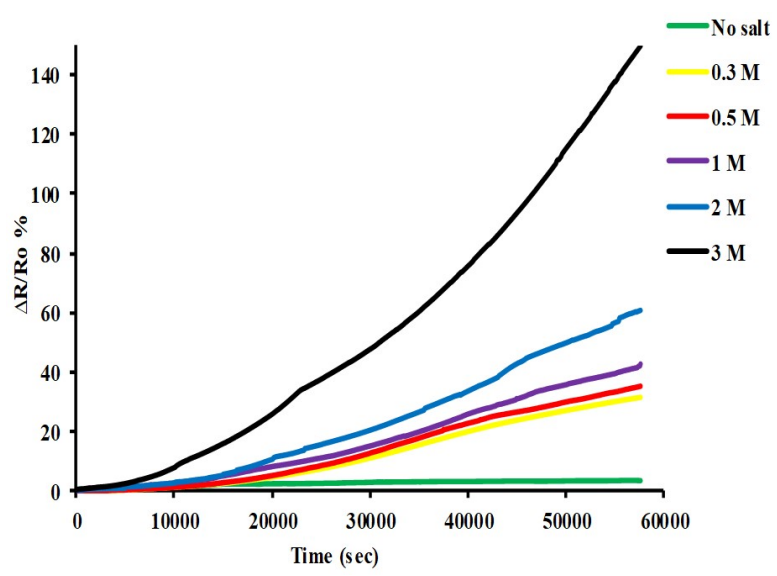

Figure 2: Normalized electrical resistance versus time for the ITO/Ag-alloy/ITO film during immersion in different $\mathrm{NaCl}$ concentration solutions. 


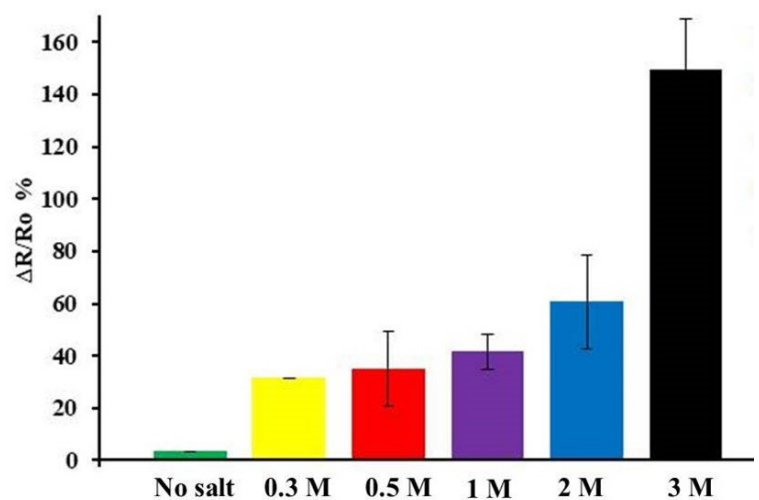

Figure 3: The effect of various concentrations on normalized electrical resistance of ITO/Ag-alloy/ITO film after $57600 \mathrm{~s}$.

until around $15000 \mathrm{~s}$ and then an increase is noticed. After $57600 \mathrm{~s}$ of immersion, the change in the electrical resistance was $31.4 \%$ for $0.3 \mathrm{M}$ and $35.2 \%$ for $0.5 \mathrm{M}$, as shown in Figure 3. For samples immersed in $2 \mathrm{M} \mathrm{NaCl}$, a higher increase in the electrical resistance was observed, indicating faster corrosion of the ITO/Ag/ITO films as compared to the samples immersed in the lower $\mathrm{NaCl}$ concentrations. After $57600 \mathrm{~s}$, a $\sim 60.7 \%$ resistance increase is observed (Figure 3) The electrical resistance trace of ITO/Ag-alloy/ITO when exposed to $3 \mathrm{M} \mathrm{NaCl}$ shows that a higher concentration of $\mathrm{NaCl}$ makes an ITO/Ag/ITO coating susceptible to a more rapid corrosion process. After $57600 \mathrm{~s}$ of experimental testing, the increase in the electrical resistance is observed to be
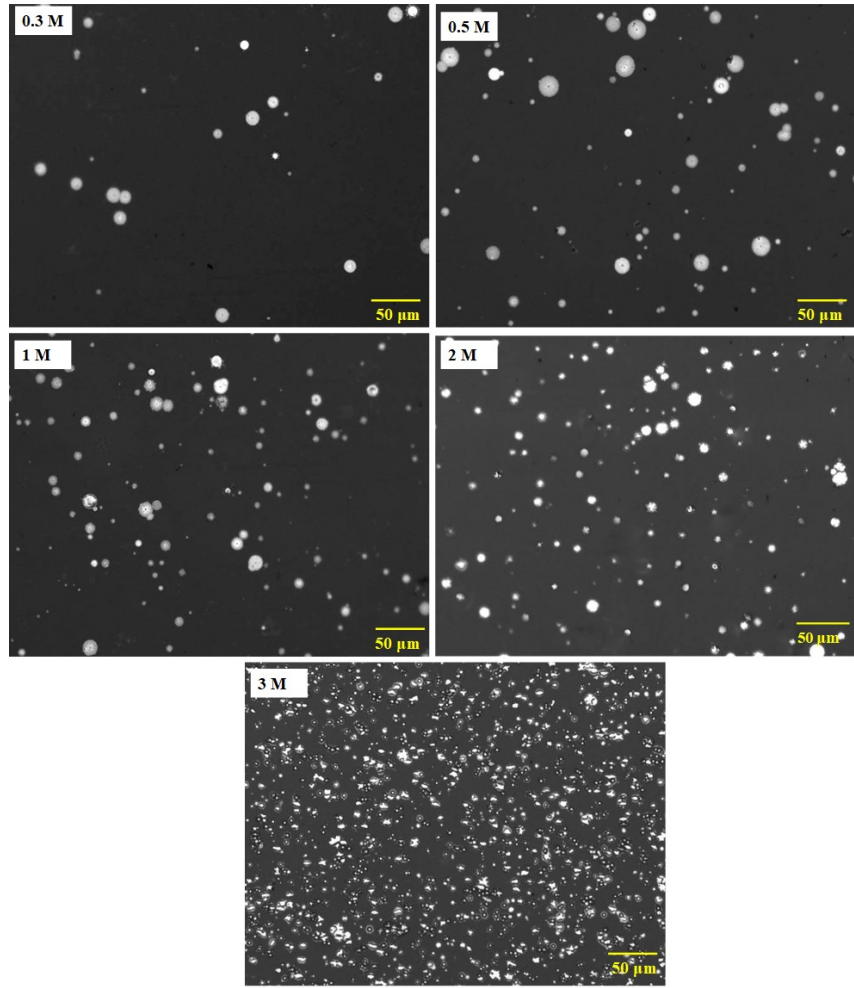

Figure 4: CLSM images of the surfaces of ITO/Ag-alloy/ITO immersed in $\mathrm{NaCl}$ of various concentrations for $57600 \mathrm{~s}$. $\sim 149.5 \%$ (Figure 3). We anticipate that, if the experiment is conducted for a significantly longer period, the ITO/Agalloy/ITO film would experience total corrosion leading to a catastrophic electrical conductivity decrease.

Figure 4 displays the progressive morphological degradation for ITO/Ag/ITO immersed in $\mathrm{NaCl}$ of various concentrations for a total of $57600 \mathrm{~s}$. The samples immersed in 0.3 $\mathrm{M}$ were degraded, showing a number of white spots. The samples that were immersed in $0.5,1,2$, and $3 \mathrm{M}$ exhibited less degradation, although they exhibited a number of white spots in places. The samples immersed in $3 \mathrm{M}$ were more degraded in comparison to the samples immersed in $0.3,0.5$, 1 , and $2 \mathrm{M}$ of the solutions, and a high number of corrosion spots was observed covering almost the whole surface of the sample. These observations agree with results in term of the electrical resistance changes over time obtained for all concentrations.

The corrosion mechanisms have been studied by Koike et al. [15], who suggested that the chloride ions $\left(\mathrm{Cl}^{-}\right)$attacked the ITO surface, passed through the top ITO layer, and reached the Ag layer. Then, the Ag atoms aggregate around the chlorine atoms to form the white clusters. Besides that, in our work, the pre-existing defects and scratches on the surface resulting from the deposition process and form handling were found to serve as nucleation points of the corrosion products (or Ag agglomerations) which appeared as white areas on the CLSM image in Figure 5. Defects and scratches in the layer are believed to facilitate the movement of $\mathrm{Cl}^{-}$to reach the $\mathrm{Ag}$ layer and enhance the agglomeration (a)

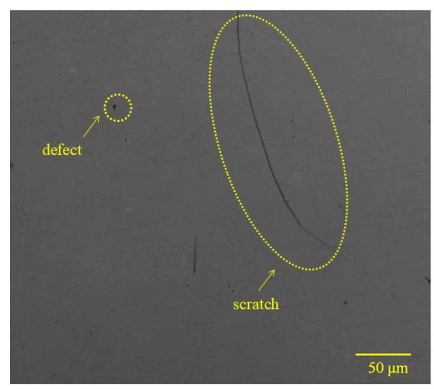

(b)

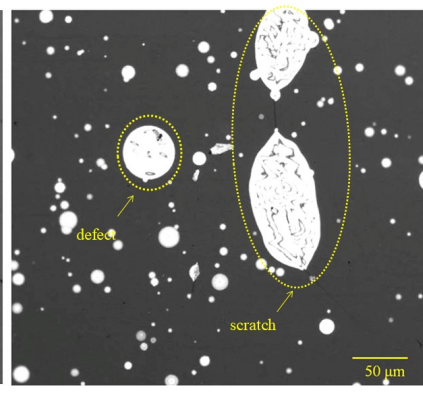

Figure 5: CLSM images showing the coating defects and scratches on the ITO/Ag-alloy/ITO surfaces before (a) and after (b) immersion in $0.5 \mathrm{M}$ for $57600 \mathrm{~s}$. (a)

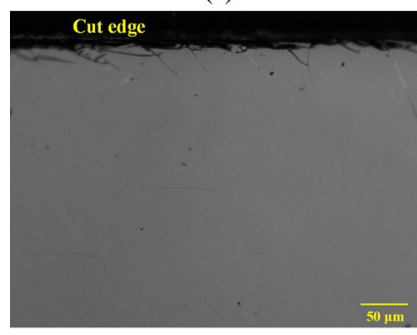

(b)

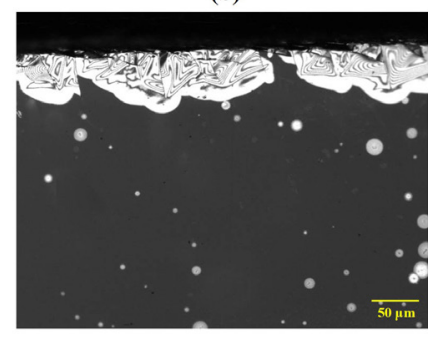

Figure 6: CLSM images showing the edge defects initiated upon cutting of ITO/Ag-alloy/ITO before (a) and after (b) immersion in $0.5 \mathrm{M}$ for $57600 \mathrm{~s}$. 
of Ag.

Apart from $\mathrm{Cl}^{-}$penetration through permeable defects or scratches on the coating, the $\mathrm{Cl}^{-}$ions could also penetrate through small cracks formed at the edges after sample cutting, as shown in Figure 6. This implies that, during cutting of specimens or fabricating of patterned electrodes in the photolithography process, care must be taken to enhance edge resolution and fidelity and to avoid edge-generated

(a)

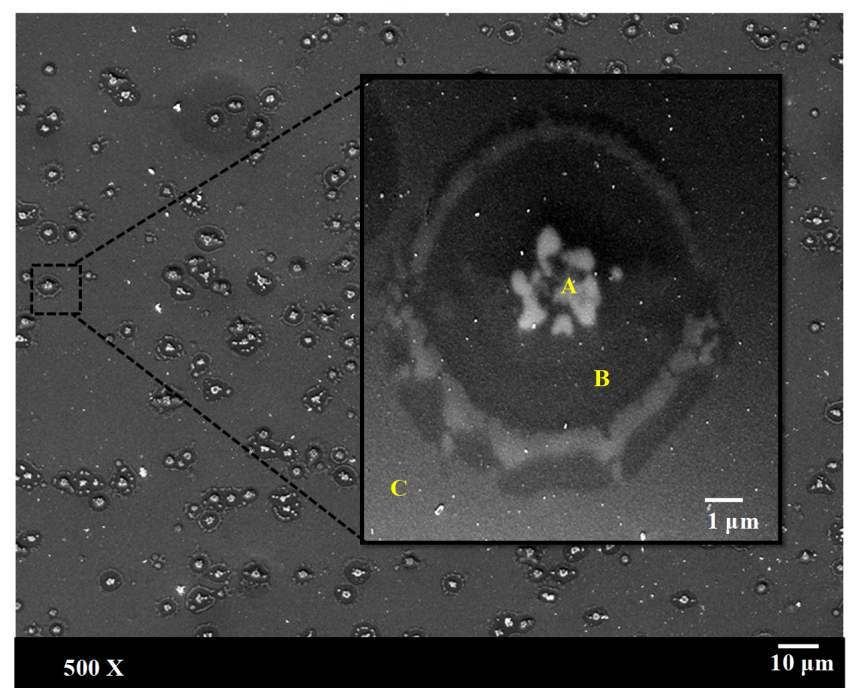

(b)

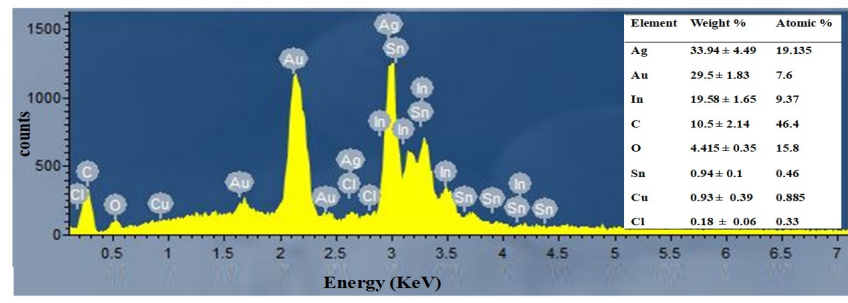

(c)

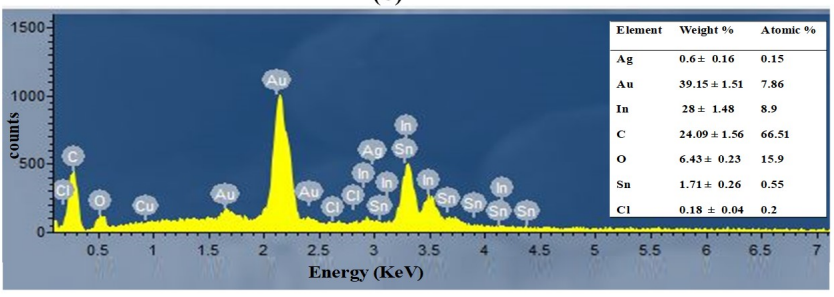

(d)

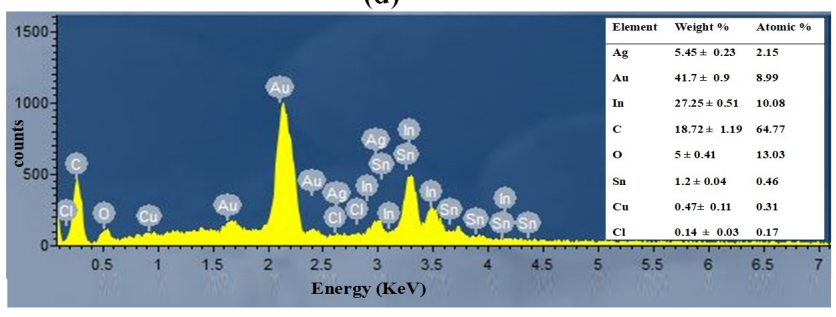

Figure 7: (a)SEM micrograph of the ITO/Ag-alloy/ITO surface immersed in the 3-M NaCl solution for 57600 seconds. (b-d) EDS spectra obtained from regions A (b), B (c), and C (d) indicated in panel (a). Inset is the table showing the percentage of each component in the film.
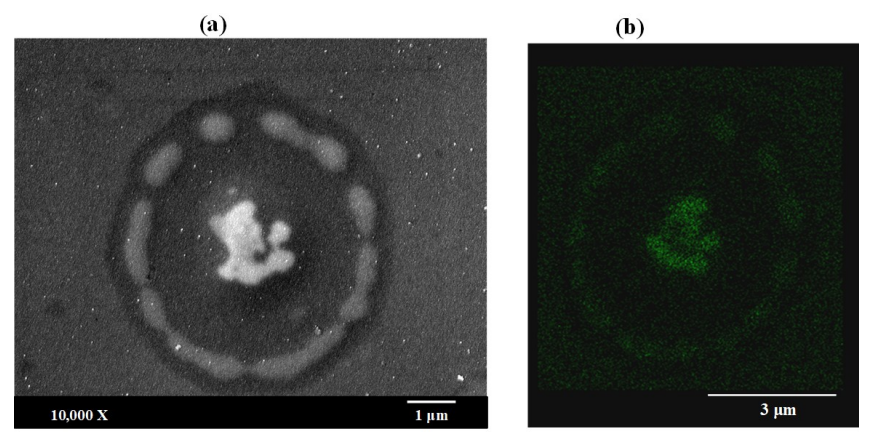

Figure 8: (a) SEM micrograph of the ITO/Ag-alloy/ITO surface after $57600 \mathrm{~s}$ submerged in the $3-\mathrm{M} \mathrm{NaCl}$ solution. (b) EDS mapping image of Ag.

micro-cracks. This will diminish corrosion spots at the edges. Such enhancement could be accomplished by edge lamination for example.

The samples immersed in $3 \mathrm{M} \mathrm{NaCl}$ were also investigated using EDS to clarify the corrosion product elements for the ITO/Ag-alloy/ITO film. EDS spot-scan analysis of surface failures and elemental mapping analysis are shown in Figures 7 and 8. The cluster of bright particles in the center of the image in region A is Ag-rich and slightly depleted in In and Sn. Along the circular edge of area in region B, there are periodic agglomerations that are rich in $\mathrm{Ag}$, but still exhibiting higher concentrations of In and Sn. In all areas of the sample, no removal of In and Sn was detectable, i.e., the $\mathrm{Sn} / \mathrm{In}$ ratio of the ITO film at region A is $\sim 0.044$, which is similar to that in the unaffected area (region $\mathrm{C}$ ), suggesting that both top and bottom ITO layers have not been removed. Eight other damage spots have been examined and yielded similar results. The high carbon content is most likely due to the PET substrate.

A tiny amount of $\mathrm{Cu}$ was also found in regions $\mathrm{A}$ and $\mathrm{C}$ and is most likely due to the Ag alloy constituent. The $\mathrm{Cu}$ content in region $\mathrm{A}$ is $0.93 \pm 0.39 \mathrm{wt} \%$, which is higher than that in other regions. Cho and Lee [21] investigated the role of $\mathrm{Cu}$ additions in the $\mathrm{Ag}$ alloy deposited between layers of indium zinc oxide and found that $\mathrm{Cu}$ did not improve the stability of the Ag-alloy layer but did improve thermal stability.

The samples immersed in 0.3 and $3 \mathrm{M} \mathrm{NaCl}$ for $57600 \mathrm{~s}$ were investigated using AFM to characterize the corrosion spot topography of the ITO/Ag-alloy/ITO film. As the three-dimensional AFM micrographs in Figure 9(a) demonstrate, the damage area for a sample immersed in the $0.3-\mathrm{M}$ solution consists primarily of circular blisters that appear to form folds in the top ITO layer, indicating interlayer delamination. The Ag layer was clustered, and its clustering, or agglomeration, was clearly seen all over the damaged area, even though the agglomeration of the Ag film is thick at the center and the height of the agglomeration at the center area is about $175 \mathrm{~nm}$, as the cross-sectional AFM micrograph shows in Figure 9(b). This indicates that the Ag agglomerations appear to be mobile once they are formed. However, when a sample is exposed to the 3-M solution, most of $\mathrm{Ag}$ 


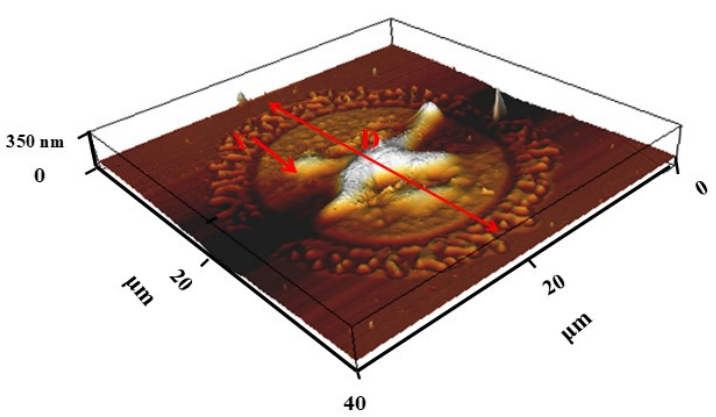

(a)

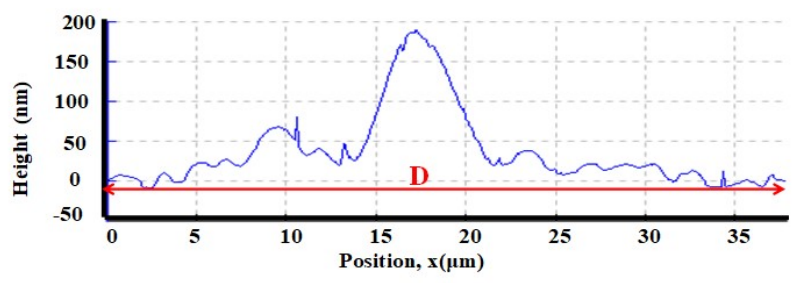

(b)

Figure 9: (a) Three-dimensional AFM image of the damage area and (b) a corresponding extracted height profile scanned across the profile line for the ITO/Ag-alloy/ITO film sample submerged in the $0.3-\mathrm{M} \mathrm{NaCl}$ solution for $57600 \mathrm{~s}$. A red line and a red arrow in panel (a) indicate the positions of the extracted profile and the $x$ direction.

has agglomerated at the center point of the circle with th height of around $80 \mathrm{~nm}$ with some parts appearing along the circumference with a height almost equals to $15 \mathrm{~nm}$ [Figure 9(b)].

Furthermore, it is observed from Figures 9(b) and 10(b) that the spot size decreases significantly with increasing the solution concentration; the spots with approximately 37 and $9 \mu \mathrm{m}$ in diameter were observed for 0.3 and $3 \mathrm{M}$, respectively. This is because the diluted solutions can not be sustained, allowing the growth into the spot region to continue, whereas thicker solutions allow a deeper attack in the small spot region.

Figure 11 exhibits the transmittance curves of ITO/Agalloy/ITO films immersed in various concentrations of $\mathrm{NaCl}$ for $57600 \mathrm{~s}$. For comparison, the transmittance of the as-received ITO/Ag-alloy/ITO thin film is also presented. As a general trend, the transmittance of the ITO/Ag-alloy/ITO films after being submerged in the $\mathrm{NaCl}$ solutions was compared to that measured for the as-received specimen. In particular, the transmittance of ITO/Ag-alloy/ITO significantly decreased after being immersed in $3 \mathrm{M} \mathrm{NaCl}$. This is likely due to the existence of several corrosion spots (Ag agglomeration) on the surface which cause surface roughening and an increase in the scattering of the incident light [9]. Similar observations have also been reported by Sahu et al. [22] on Ag films exposed to $200^{\circ} \mathrm{C}$ for $30 \mathrm{~min}$. They attributed the decrease in transmittance of the Ag film to the agglomeration of $\mathrm{Ag}$ and consequently to the enhanced scattering of the light. These results are also consistent with

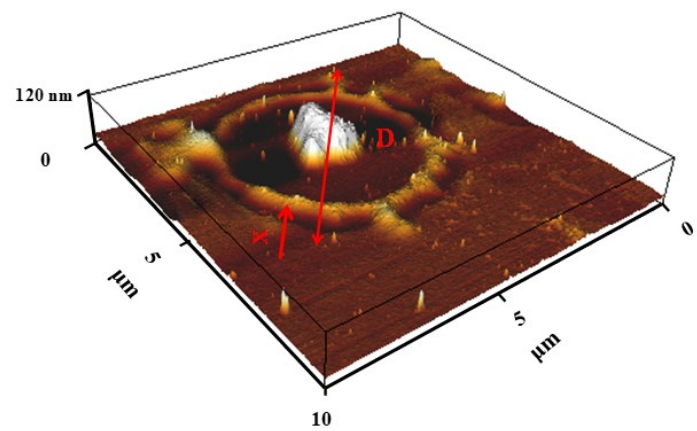

(a)

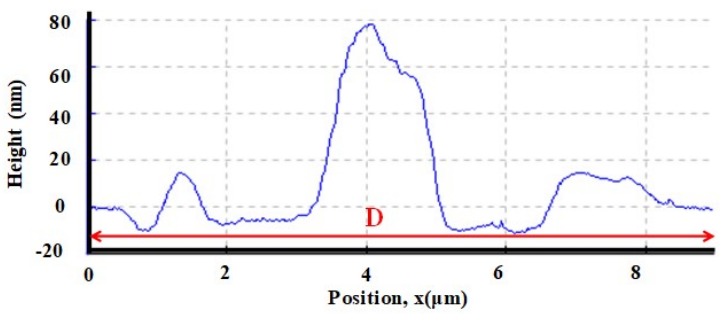

(b)

Figure 10: Same as Figure 9, but the sample was immersed in the 3-M NaCl solution.

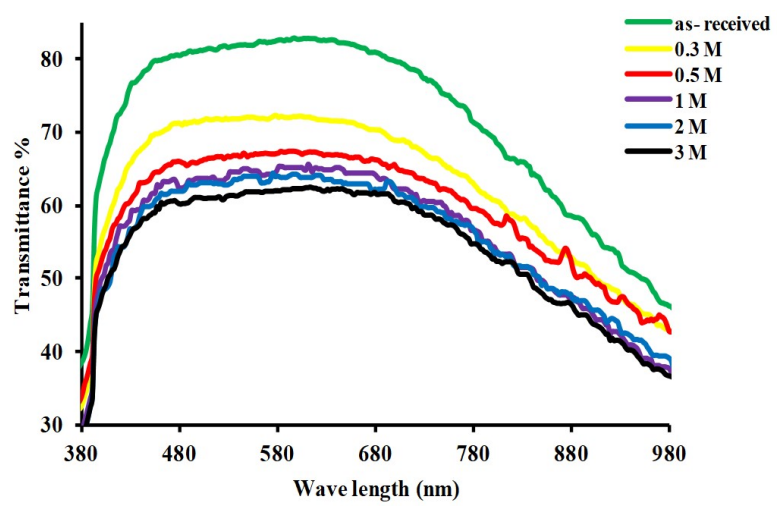

Figure 11: The effect of various concentrations on optical transmittances of the flexible ITO/Ag-alloy/ITO film after $57600 \mathrm{~s}$.

those observed previously by Koike et al. [23], who found that the electrical sheet resistance was increased significantly and the transmittance was significantly decreased in the multilayer sputter coated ITO (40 nm)/Ag (10 nm)/ITO (40 $\mathrm{nm}$ )/glass during a 22-h immersion test in an aqueous $\mathrm{NaCl}$ solution.

\section{B. Combined corrosion and fatigue degrada- tion of ITO/Ag-alloy/ITO}

To investigate the effect of repeated loading on the structural integrity of the ITO/Ag-alloy/ITO-coated PET substrates under the fatigue and fatigue-corrosion tests, the electrical resistance changes were recorded in situ. The tests were run at a frequency of $2 \mathrm{~Hz}$ as this is the highest frequency relevant to the roll-to-roll manufacturing process and in-service conditions. The combined fatigue-corrosion ex- 


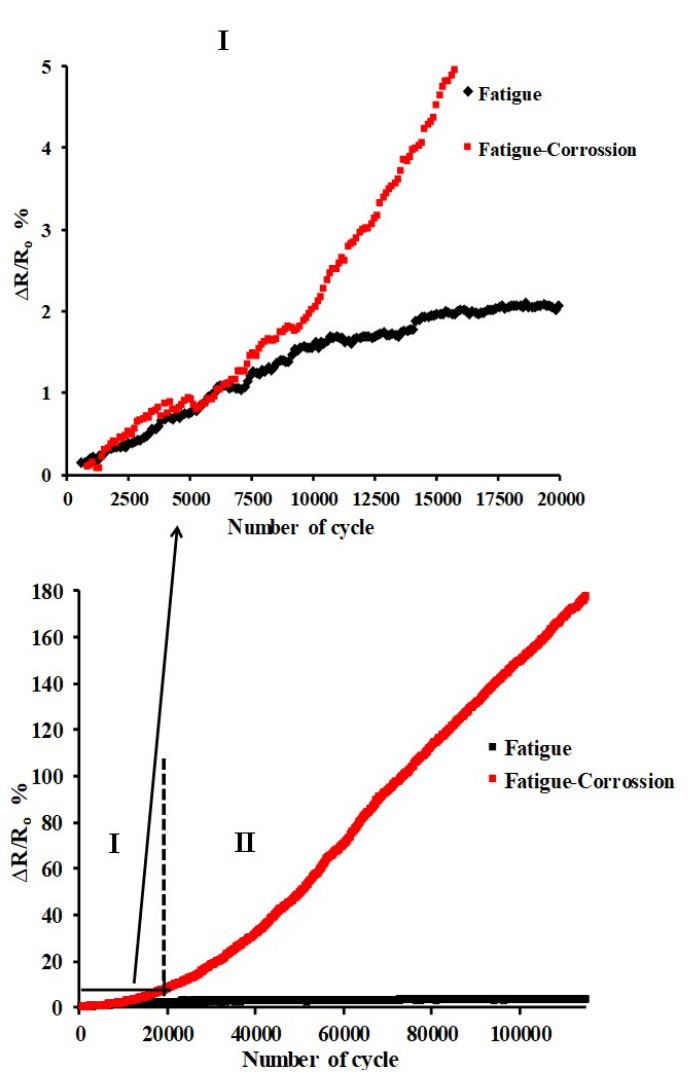

Figure 12: Normalized electrical resistance versus the number of cycles of ITO/Ag-alloy/ITO under bending fatigue and bending fatigue-corrosion conditions. The upper inset represents the enlarged result up to 2000 cycles, which clearly reflects the initial increase of the electrical resistance.

periment was performed in $0.3 \mathrm{M} \mathrm{NaCl}$ since the time to corrode ITO/Ag-alloy/ITO is adequate to allow meaningful observations to be made. Using data from the bending tests, a mandrel diameter of $25 \mathrm{~mm}$, which is equivalent to a tensile strain of $0.5 \%$, was selected for the fatigue tests.

Figure 12 shows the percentage increase in the electrical resistance as a function of the number of cycles under fatigue and fatigue-corrosion. The mechanism of bending fatigue appears to be similar to that observed in our previous work for the twisting fatigue test [24], and the observed increase in the electrical resistance can be split into two distinct regions. In the first region (region I), the increment in the normalized electrical resistance is associated with the dimensional change of the compliant substrate until an equilibrium size is attained [25]. In the second region (region II), the gradually increasing resistance is likely to be due to cracking of the ITO/Ag-alloy/ITO film.

The mechanism of corrosion-fatigue in region I is similar to the fatigue-only case. However, in region II, the high rate of the electrical resistance increase was observed (Figure 12). For example, at the same applied strain of $0.5 \%$ and 115200 cycles, the sample under fatigue-corrosion exhibited an increase of the resistance of $\sim 177 \%$, which is significantly higher than that of $\sim 3.4 \%$ for the sample under fatigue only.

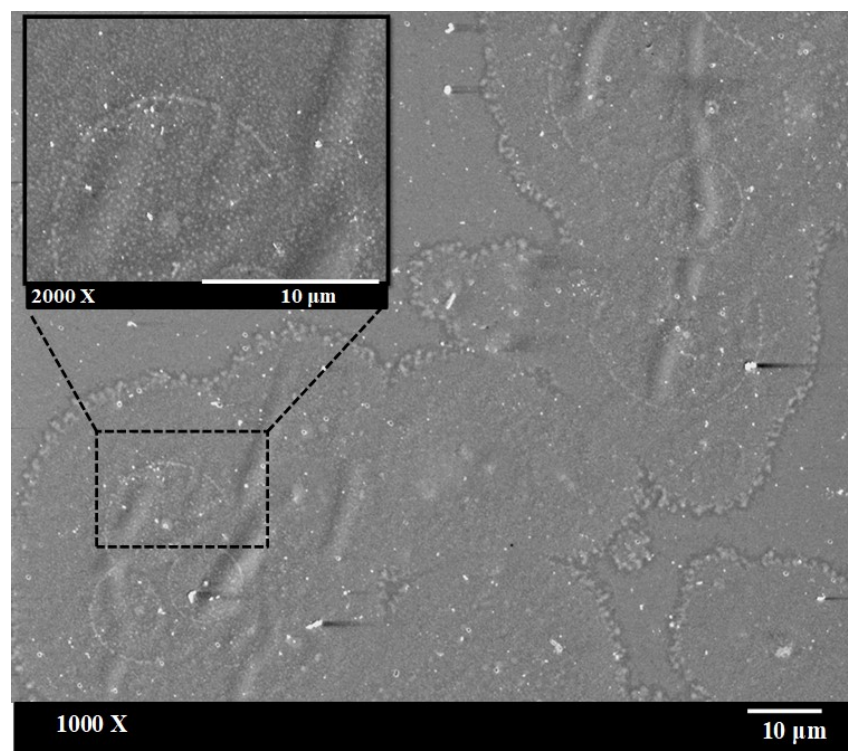

Figure 13: SEM image with an enlarged image of the surface of the ITO/Ag-alloy/ITO film after the fatigue-corrosion test for 115200 cycles.

Such phenomena may be attributed to the combined action of mechanical cracking and corrosion with the contribution of corrosion to increase the electrical resistance being significant.

Bejitual [26] reported that, in the bending fatigue tests on a single ITO $(200 \mathrm{~nm})$ film coated on a PET substrate, the change in the electrical resistance was $50 \%$ at 150000 cycles for $0.6 \%$ strain. This means that the ITO/Ag-alloy/ITO film shows superior flexibility in compression to a single ITO layer. This is attributed to the presence of the ductile Ag middle layer. However, a direct comparison of corrosionfatigue (salt immersion) reliability of ITO/Ag-alloy/ITO with single ITO film could not be made due to lack of data available in the literature.

Cracking was not observed on the surface of the samples after fatigue. However, some submicron cracks may have probably formed on the ITO/Ag-alloy/ITO films and were associated with a slight increase of the electrical resistance with increasing the number of cycles. Then, upon load removal, strain recovery in the polymer results in the closure of the cracks and leads them to be not visible [27], in a similar manner to that in the twisting fatigue test at low angles. Figure 13 presents an SEM image of the ITO/Ag-alloy/ITO samples after corrosion-fatigue. No crack was visible, possibly because of the same reason as mentioned above in the fatigue-only case. Ag agglomeration is observed to spread inside the circular spot.

At the area where circular-blisters exists, damages such as wrinkles or buckling features were observed. This is because the externally applied mechanical stress which induces stress in the thin film combined with mechanical energy (through surface tension and capillary forces provided by the solution) can cause buckling [28].

This suggests that the presence of the aqueous $\mathrm{NaCl}$ solu- 
tion together with mechanical stress promotes electrical failure. Therefore, the effect of mechanical strain coupled with corrosion must be taken into account for the durability and lifetime prediction of ITO/Ag-alloy/ITO electrodes.

\section{CONCLUSION}

The corrosion behavior of the ITO/Ag-alloy/ITO film coated PET substrate in the presence of $0.3,0.5,1,2$, and 3 $\mathrm{M}$ aqueous solutions of $\mathrm{NaCl}$ was studied by in situ electrical measurements to understand its stability when in use in aggressive environments. It was observed that the rapid corrosion of the ITO/Ag-alloy/ITO film coating is promoted by high $\mathrm{NaCl}$ concentration and that was associated with observations of the surface of the films. Furthermore, it is shown that the presence of defects in the deposited films can provide a driving force to form the $\mathrm{Ag}$ agglomerations which, in turn, degrade the conductivity of the ITO/Ag-alloy /ITO film. This suggests that defects in the film should be minimized to avoid excessive corrosion. Moreover, the ITO/ Ag-alloy/ITO film submerged in high salt concentration such as 1,2 , and $3 \mathrm{M}$ exhibited high degradation of optical transmittance. This is because an increase in chloride concentration of the solution results in a higher density of spots on the surface which causes severe light scattering. In the cyclic bending fatigue tests under the aggressive salt environments, the increase in the normalized electrical resistance of the samples under corrosion-bending fatigue test was significantly higher than that under bending fatigue alone. This indicates that the ITO/Ag-alloy/ITO film failure is dominated by corrosion, while the external mechanical stress is an assisting failure mechanism. The present study can be proved particularly useful information when designing flexible devices that need to perform in aggressive environments.

\section{Acknowledgments}

The authors would like to acknowledge financial support from the Kurdistan Regional Government HCDP program with grant number DD-07. We would also like to thank Mr. Frank Biddlestone for his technical support. Providing samples from Dr. G. Potoczny is gratefully acknowledged.

\section{References}

[1] K. A. Sierros, N. J. Morris, S. N. Kukureka, and D. R. Cairns, Wear 267, 625 (2009).

[2] M. M. Hamasha, K. Alzoubi, S. Lu, and S. B. Desu, Thin Solid Films 519, 6033 (2011).

[3] K. Leppänen, B. Augustine, J. Saarela, R. Myllylä, and T. Fabritius, Sol. Energy Mater. Sol. Cells 117, 512 (2013).

[4] Q. Xu, W. Shen, Q. Huang, Y. Yang, R. Tan, K. Zhu, N. Dai, and W. Song, J. Mater. Chem. C 2, 3750 (2014).

[5] M. Girtan, Sol. Energy Mater. Sol. Cells 100, 153 (2012).

[6] B. Szyszka, in: Handbook of Visual Display Technology, edited by J. Chen, W. Cranton, and M. Fihn (Springer, Cham, 2016) pp. 1271-1285.
[7] X. T. Hao, F. R. Zhu, K. S. Ong, and L. W. Tan, Semicond. Sci Technol. 21, 19 (2005).

[8] C. Guillén and J. Herrero, Sol. Energy Mater. Sol. Cells 92, 938 (2008).

[9] C. Guillén and J. Herrero, Thin Solid Films 520, 1 (2011).

[10] C.-T. Chu, P. D. Fuqua, and J. D. Barrie, Appl. Opt. 45, 1583 (2006).

[11] E. Ando, S. Suzuki, N. Aomine, M. Miyazaki, and M. Tada, Vacuum 59, 792 (2000).

[12] G. Folcher, H. Cachet, M. Froment, and J. Bruneaux, Thin Solid Films 301, 242 (1997).

[13] F. C. Krebs, S. A. Gevorgyan, and J. Alstrup, J. Mater. Chem. 19, 5442 (2009).

[14] S. J. Nadel, J. Vac. Sci. Technol. A 5, 2709 (1987).

[15] K. Koike, F. Yamazaki, T. Okamura, and S. Fukuda, J. Vac.

Sci. Technol. A 25, 527 (2007).

[16] S.-W. Chen, C.-H. Koo, H.-E. Huang, and C.-H. Chen, Mater. Trans. 46, 2536 (2005).

[17] K. A. Sierros, N. J. Morris, K. Ramji, and D. R. Cairns, Thin Solid Films 517, 2590 (2009).

[18] D. W. Mohammed, Mechanical and Electro-mechanical Reliability of Transparent Oxide Thin Films for Flexible Electronics Applications, Ph.D. thesis, University of Birmingham, 2016.

[19] T. S. Bejitual, D. Compton, K. A. Sierros, D. R. Cairns, and S. N. Kukureka, Thin Solid Films 528, 229 (2013).

[20] Z. Chen, B. Cotterell, and W. Wang, Eng. Fract. Mech. 69, 597 (2002).

[21] S.-H. Cho and W.-J. Lee, Jpn. J. Appl. Phys. 49, 111102 (2010).

[22] D. R. Sahu and J.-L. Huang, Mater. Sci. Eng. B 130, 295 (2006).

[23] K. Koike, K. Shimada, and S. Fukuda, Corros. Sci. 51, 2557 (2009).

[24] D. W. Mohammed, R. B. Ameen, K. A. Sierros, J. Bowen, and S. N. Kukureka, Thin Solid Films 645, 241 (2018).

[25] D. R. Cairns and G. P. Crawford, Proc. IEEE 93, 1451 (2005).

[26] T. S. Bejitual, Reliability of flexible optoelectronic device electrodes, Ph.D. thesis, West Virginia University, 2013.

[27] D. Nicholls, Physical ageing in semi-crystalline polyethylene terephthalate, Master's thesis, University of Birmingham, 2009.

[28] A. A. Volinsky and P. Waters, in: Mechanical Self-Assembly: Science and Applications, edited by X. Chen (Springer, New York, 2013) Chap. 8.

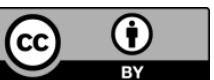

All articles published on e-J. Surf. Sci. Nanotechnol. are licensed under the Creative Commons Attribution 4.0 International (CC BY 4.0). You are free to copy and redistribute articles in any medium or format and also free to remix, transform, and build upon articles for any purpose (including a commercial use) as long as you give appropriate credit to the original source and provide a link to the Creative Commons (CC) license. If you modify the material, you must indicate changes in a proper way.

Published by The Japan Society of Vacuum and Surface Science 